Research Article

\title{
Global Adaptive Control of Stochastic Nonlinear Systems with Linearly Bounded Unmeasurable States by Output Feedback
}

\author{
Qiangde Wang and Chunling Wei \\ Institute of Automation, Qufu Normal University, Rizhao 276826, China \\ Correspondence should be addressed to Qiangde Wang,wqdwchl@sohu.com
}

Received 11 November 2011; Revised 30 January 2012; Accepted 13 February 2012

Academic Editor: Rafael Martinez-Guerra

Copyright (C) 2012 Q. Wang and C. Wei. This is an open access article distributed under the Creative Commons Attribution License, which permits unrestricted use, distribution, and reproduction in any medium, provided the original work is properly cited.

The problem of the output feedback stochastic stabilization is investigated for a class of stochastic nonlinear systems with linearly bounded unmeasurable states. Under the condition that the inverse dynamics is stochastic input-to-state stable and the nonlinear functions satisfy the linear growth conditions with unknown growth rate, an adaptive output feedback controller is proposed to make the closed-loop system globally stable in probability and the states of the closed-loop system converge to zero almost surely. A simulation example is provided to show the effectiveness of the theoretical results.

\section{Introduction}

The output feedback control for stochastic nonlinear systems is a challenging research subject in control theory. Since the design of output feedback control is more difficult and more practical in engineering than that of full-state feedback control, many output feedback controller design schemes for stochastic nonlinear systems have been proposed [1-8]. For the deterministic nonlinear systems, some counterexamples were given in [9] to indicate that the global output feedback stabilization of the nonlinear systems is usually impossible unless some growth conditions on the unmeasurable states of the systems are introduced. From then on, the problems of global output feedback stabilization for nonlinear systems with various growth conditions were studied [10-15]. Recently, some output feedback controllers were designed for the stochastic nonlinear systems in which nonlinear terms depend on the output and unmeasurable inverse dynamics or unmodeled dynamics [3, 16-19]; moreover, an output feedback controller was proposed for the stochastic nonlinear systems with the nonlinear terms dependent on the unmeasurable states and unmeasurable inverse dynamics [20]. However [20] considers only the case that the parameters in the bounding functions 
are known; when they are unknown constants, the global output feedback control of the stochastic system becomes much more difficult due to the lack of effective observer design techniques [21]. Recently, the output feedback stabilization for a class of stochastic nonlinear systems with stochastic iISS inverse dynamics was studied in [22-27].

In this paper, we focus on the output feedback stabilization for a class of stochastic nonlinear systems in which the drift and diffusion terms are dependent on unmeasurable states besides the output and unmeasurable inverse dynamics and without knowing the growth rate. Under the assumption that the inverse dynamics are SISS (stochastic input-tostate stable), an adaptive output feedback controller with a high-gain observer is constructed to make the closed-loop system globally stable in probability and the states of the closedloop converge to zero almost surely. The contribution of this paper consists of two aspects. (i) By adopting the universal adaptive output feedback controller presented in [21], the output feedback control problem of stochastic nonlinear system with the nonlinear functions being bounded with unknown constants is investigated. (ii) The two assumptions in [20] are relaxed (see Remark 3.3).

The remained of this paper is organized as follows. Section 2 provides some preliminaries and Section 3 gives the problem to be studied. An adaptive output feedback controller is proposed in Section 4. The main results of this paper and the stability analysis of the closedloop system are presented in Section 5. Section 6 gives a simulation example to demonstrate the correctness of the theoretical results and some concluding remarks are contained in Section 7.

\section{Preliminaries}

Consider the following stochastic nonlinear systems:

$$
d x=f(x, t) d t+g(x, t) d w,
$$

where $x \in R^{n}$ is the state, $w$ is an $m$-dimensional standard Wiener process defined on the complete probability space $(\Omega, \mathcal{F}, P)$ with $\Omega$ being a sample space, $\mathcal{F}$ being a $\sigma$-field, and $P$ being the probability measure, and $f: R^{n} \times R_{+} \rightarrow R^{n}$ and $g: R^{n} \times R_{+} \rightarrow R^{m}$ are locally Lipschitz continuous functions in $x$ uniformly in $t$ and satisfy the linear growth condition. Meanwhile, we assume that $f(0, t)=0$ and $g(0, t)=0$.

Throughout this paper, we define the differential operator $\mathfrak{L}$ as follows:

$$
\mathfrak{L} V=\frac{\partial V}{\partial t}+\frac{\partial V}{\partial x} f(x, t)+\frac{1}{2} \operatorname{Tr}\left(g^{T} \frac{\partial^{2} V}{\partial x^{2}} g\right)
$$

where $V(x, t) \in C^{2,1}\left(R^{n} \times R_{+}, R_{+}\right)$. We also denote by $D\left(R_{+} ; R_{+}\right)$the family of all functions $\gamma: R_{+} \rightarrow R_{+}$such that $\int_{0}^{+\infty} \gamma(t) d t<\infty$.

The following lemma provides a sufficient condition to ensure the existence and uniqueness of global solution for the system (2.1). 
Lemma 2.1. For system (2.1), assume that both terms $f(x, t)$ and $g(x, t)$ are locally Lipschitz, and $f(0, t)$ and $g(0, t)$ are bounded uniformly in $t$. If there exists a function $V(x, t) \in C^{2,1}\left(R^{n} \times R_{+}, R_{+}\right)$ such that for a constant $K>0$ and any $t \geq 0$,

$$
\begin{gathered}
\mathfrak{L} V(x, t) \leq K(1+V(x, t)), \\
\liminf _{|x| \rightarrow \infty} V(x, t)=\infty,
\end{gathered}
$$

then, there exists a unique solution on $[0, \infty)$ for any initial value $x(0)=x_{0} \in R^{n}$.

Proof. This is a special form of Theorem 1 in [28].

Lemma 2.2. For system (2.1), assume that there exist functions $V(x, t) \in C^{2,1}\left(R^{n} \times R_{+}, R_{+}\right), \mu_{1} \in$ $\mathcal{K}, \mu_{2} \in \mathcal{K}, \mu_{3} \in \mathcal{K}$, and $\gamma(t) \in D\left(R_{+} ; R_{+}\right)$such that

$$
\begin{gathered}
\mu_{1}(x) \leq V(x, t) \leq \mu_{2}(x), \\
\mathfrak{L} V(x, t) \leq \gamma(t)-\mu_{3}(x),
\end{gathered}
$$

for all $(x, t) \in\left(R^{n}, R_{+}\right)$. Then, for any initial value $x(0)=x_{0} \in R^{n}$, the solution of system (2.1) is bounded almost surely and has the properties that

$$
\begin{gathered}
\lim _{t \rightarrow \infty}\left\|x\left(t ; x_{0}\right)\right\|=0 \text { a.s., } \\
\int_{0}^{+\infty}(\gamma(t)-\mathfrak{L} V(x, t)) d t<+\infty \text { a.s. }
\end{gathered}
$$

Proof. This Lemma can be proved by using Theorem 2.6 in [29] and Corollary 4.2 in [30].

\section{Problem Statement}

Consider the following stochastic system:

$$
\begin{aligned}
d \eta & =f_{0}(\eta, y, t) d t+g_{0}(\eta, y, t) d w \\
d x_{1} & =\left(x_{2}+f_{1}(x, \eta, t)\right) d t+g_{1}(x, \eta, t) d w \\
\vdots & \\
d x_{n-1} & =\left(x_{n}+f_{n-1}(x, \eta, t)\right) d t+g_{n-1}(x, \eta, t) d w \\
d x_{n} & =\left(u+f_{n}(x, \eta, t)\right) d t+g_{n}(x, \eta, t) d w \\
y & =x_{1}
\end{aligned}
$$

where $\eta \in R^{m}$ is the state of the unmeasurable inverse dynamics, $x=\left(x_{1}, \ldots, x_{n}\right)$ is the state, $u \in R$ is the control input, $y \in R$ is the measured output, $f_{0} \in R^{m}, g_{0} \in R^{m \times s}, f_{i} \in R$, and $g_{i}^{T} \in R^{s}, i=1, \ldots, n$ are uncertain locally Lipschitz in the first two arguments uniformly in $t$. Moreover $f_{i}(0,0, t)=0, g_{i}(0,0, t)=0, i=0, \ldots, n$, and $w$ is $m$-dimensional standard Wiener process as defined in Section 2. For system (2.1), we give the following assumptions. 
Assumption 3.1. There exist unknown positive constants $\theta_{1}, \theta_{2}, \theta_{3}$, and $\theta_{4}$ such that

$$
\begin{array}{rr}
\left|f_{i}(x, \eta, t)\right| \leq \theta_{1}\left(\left|x_{1}\right|+\cdots+\left|x_{i}\right|\right)+\theta_{2}\|\eta\|, & i=1, \ldots, n, \\
\left\|g_{i}(x, \eta, t)\right\| \leq \theta_{3}\left(\left|x_{1}\right|+\cdots+\left|x_{i}\right|\right)+\theta_{4}\|\eta\|, & i=1, \ldots, n .
\end{array}
$$

Assumption 3.2. There exist unknown positive constants $\alpha$ and $r$ and a positive definite function $V_{\eta}(\eta, t)$ which is radially unbounded in $\eta$ uniformly in $t$, such that

$$
\mathfrak{L} V_{\eta} \leq-\alpha\|\eta\|^{2}+r|y|^{2}
$$

Remark 3.3. In [20] (B2 and B3), the positive constants $\theta_{1}, \theta_{2}, \theta_{3}, \theta_{4}, \alpha$, and $r$ are known, but they are unknown in this paper. Thus, it is more difficult and meaningful to design an output feedback stabilizing control law for system (3.1).

The control problem in this paper is precisely formulated as follows.

For system (3.1), under Assumptions 3.1 and 3.2, the control objective is to design a smooth dynamic output feedback controller:

$$
\begin{aligned}
& \dot{x}=\varpi(x, y), \\
& u=\mu(x, y),
\end{aligned}
$$

such that the solution of closed-loop system (3.1) and (3.4) is bounded a.s., $\lim _{t \rightarrow \infty}\|x(t)\|=$ 0 a.s., and $\lim _{t \rightarrow \infty}\|\eta(t)\|=0$ a.s.

\section{Adaptive Output Feedback Controller Design}

First, we design a state observer for system (3.1):

$$
\begin{aligned}
\dot{\hat{x}}_{1} & =\widehat{x}_{2}+L a_{1}\left(y-\widehat{x}_{1}\right) \\
\vdots & \\
\dot{\hat{x}}_{n-1} & =\widehat{x}_{n}+L^{n-1} a_{n-1}\left(y-\widehat{x}_{1}\right) \\
\dot{\hat{x}}_{n} & =u+L^{n} a_{n}\left(y-\widehat{x}_{1}\right) \\
\dot{L} & =\left(y-\widehat{x}_{1}\right)^{2}, \quad L(0)=1,
\end{aligned}
$$

where $a_{i}>0, i=1, \ldots, n$ are the coefficients of the Hurwitz polynomial $p(s)=s^{n}+a_{1} s^{n-1}+$ $\cdots+a_{n}$ 
Let $e_{i}=\left(x_{i}-\widehat{x}_{i}\right) / L^{i-1}, i=1, \ldots, n$, and $e=\left(e_{1}, \ldots, e_{n}\right)^{T}$. Then we can obtain the following error dynamic system:

$$
\begin{aligned}
d e_{1} & =\left(L e_{2}-L a_{1} e_{1}+f_{1}\right) d t+g_{1} d w \\
& \vdots \\
d e_{n-1} & =\left(L e_{n}-L a_{n-1} e_{1}+\frac{f_{n-1}}{L^{n-2}}-(n-2) \frac{\dot{L}}{L} e_{n-1}\right) d t+\frac{g_{n-1}}{L^{n-2}} d w \\
d e_{n} & =\left(-L a_{n} e_{1}+\frac{f_{n}}{L^{n-1}}-(n-1) \frac{\dot{L}}{L} e_{n}\right) d t+\frac{g_{n}}{L^{n-1}} d w .
\end{aligned}
$$

It can be written as the following compact form:

$$
d e=\left(L A e+F-\frac{\dot{L}}{L} D e\right) d t+G d w
$$

where

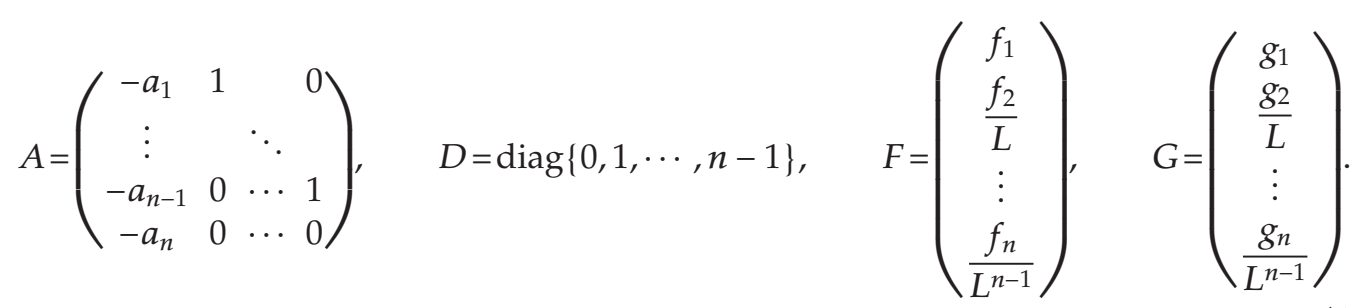

Since the polynomial $p(s)=s^{n}+a_{1} s^{n-1}+\cdots+a_{n}$ is Hurwitz, there exists a positive definite matrix $P$ such that

$$
A^{T} P+P A=-I, \quad D^{T} P+P D \geq 0 .
$$

Let $z_{i}=\widehat{x}_{i} / L^{i-1}, i=1, \ldots, n, v=u / L^{n}$, and $z=\left(z_{1}, \ldots, z_{n}\right)^{T}$. Then we can obtain

$$
\begin{aligned}
d z_{1} & =\left(L z_{2}+L a_{1} e_{1}\right) d t \\
& \vdots \\
d z_{n-1} & =\left(L z_{n}+L a_{n-1} e_{1}-(n-2) \frac{\dot{L}}{L} z_{n-1}\right) d t \\
d z_{n} & =\left(L v+L a_{n} e_{1}-(n-1) \frac{\dot{L}}{L} z_{n}\right) d t .
\end{aligned}
$$

Design the controller

$$
u=L^{n} v=-L^{n}\left(b_{1} z_{1}+\cdots+b_{n} z_{n}\right)=-\left(L^{n} b_{1} \widehat{x}_{1}+L^{n-1} b_{2} \widehat{x}_{2}+\cdots+L b_{n} \widehat{x}_{n}\right)
$$


where $b_{i}>0, i=1, \ldots, n$, are the coefficients of the Hurwitz polynomial $h(s)=s^{n}+b_{n} s^{n-1}+$ $\cdots+b_{1}$. Substituting (4.7) into (4.6), we have

$$
d z=\left(L B z+L a e_{1}-\frac{\dot{L}}{L} D z\right) d t
$$

where

$$
B=\left(\begin{array}{cccc}
0 & 1 & \cdots & 0 \\
\vdots & \vdots & \ddots & \vdots \\
0 & 0 & \cdots & 1 \\
-b_{1} & -b_{2} & \cdots & -b_{n}
\end{array}\right), \quad a=\left(a_{1}, \ldots, a_{n}\right)^{T}
$$

For the polynomial $h(s)=s^{n}+b_{n} s^{n-1}+\cdots+b_{1}$ being Hurwitz, there exists a positive definite matrix $Q$ such that

$$
B^{T} Q+Q B=-2 I, \quad D^{T} Q+Q D \geq 0 .
$$

Then the closed-loop system can be represented in the following compact form:

$$
\begin{aligned}
d \eta & =f_{0}(\eta, y, t) d t+g_{0}(\eta, y, t) d w, \\
d e & =\left(L A e+F-\frac{\dot{L}}{L} D e\right) d t+G d w, \\
d z & =\left(L B z+L a e_{1}-\frac{\dot{L}}{L} D z\right) d t, \\
\dot{L} & =\left(y-\widehat{x}_{1}\right)^{2}=e_{1}^{2} .
\end{aligned}
$$

\section{Stability Analysis of the Closed-Loop System}

By Assumption 3.1 and $L \geq 1$, we have the following estimates:

$$
\begin{aligned}
\|F\|^{2} & \leq \sum_{i=1}^{n}\left|\frac{f_{i}}{L^{i-1}}\right|^{2} \leq \sum_{i=1}^{n}\left[\frac{1}{L^{i-1}} \theta_{1}\left(\left|x_{1}\right|+\cdots+\left|x_{i}\right|\right)+\theta_{2}\|\eta\|\right]^{2} \\
& \leq 2 \sum_{i=1}^{n}\left[\frac{1}{L^{i-1}} \theta_{1}\left(\left|x_{1}\right|+\cdots+\left|x_{i}\right|\right)\right]^{2}+2 n \theta_{2}^{2}\|\eta\|^{2} \\
& \leq 2 n \theta_{1}^{2}\left[\left(\left|x_{1}\right|+\frac{\left|x_{2}\right|}{L}+\cdots+\frac{\left|x_{n}\right|}{L^{n-1}}\right)\right]^{2}+2 n \theta_{2}^{2}\|\eta\|^{2}
\end{aligned}
$$


Mathematical Problems in Engineering

$$
\begin{aligned}
& \leq 2 n \theta_{1}^{2}\left[\left(\left|e_{1}\right|+\left|\widehat{x}_{1}\right|+\left|e_{2}\right|+\frac{\left|\widehat{x}_{2}\right|}{L}+\cdots+\left|e_{n}\right|+\frac{\left|\widehat{x}_{n}\right|}{L^{n-1}}\right)\right]^{2}+2 n \theta_{2}^{2}\|\eta\|^{2} \\
& =2 n \theta_{1}^{2}\left[\left(\left|e_{1}\right|+\cdots+\left|e_{n}\right|+\left|z_{1}\right|+\cdots+\left|z_{n}\right|\right)\right]^{2}+2 n \theta_{2}^{2}\|\eta\|^{2} \\
& \leq 4 n^{2} \theta_{1}^{2}\left(\|e\|^{2}+\|z\|^{2}\right)+2 n \theta_{2}^{2}\|\eta\|^{2}, \\
\|G\|^{2} & \leq \sum_{i=1}^{n}\left|\frac{g_{i}}{L^{i-1}}\right|^{2} \leq \sum_{i=1}^{n}\left[\frac{1}{L^{i-1}} \theta_{3}\left(\left|x_{1}\right|+\cdots+\left|x_{i}\right|\right)+\theta_{4}\|\eta\|\right]^{2} \\
& \leq 2 \sum_{i=1}^{n}\left[\frac{1}{L^{i-1}} \theta_{3}\left(\left|x_{1}\right|+\cdots+\left|x_{i}\right|\right)\right]^{2}+2 n \theta_{4}^{2}\|\eta\|^{2} \\
& \leq 2 n \theta_{3}^{2}\left[\left(\left|x_{1}\right|+\frac{\left|x_{2}\right|}{L}+\cdots+\frac{\left|x_{n}\right|}{L^{n-1}}\right)\right]^{2}+2 n \theta_{4}^{2}\|\eta\|^{2} \\
& \leq 4 n^{2} \theta_{3}^{2}\left(\|e\|^{2}+\|z\|^{2}\right)+2 n \theta_{4}^{2}\|\eta\|^{2} .
\end{aligned}
$$

Select $V_{1}=\delta e^{T} P e$, where $\delta>0$ is a parameter to be specified later. Then, by (2.2), (4.5), (4.11), and (5.1) we have

$$
\begin{aligned}
\mathfrak{L} V_{1}= & \delta e^{T} L\left(A^{T} P+P A\right) e+2 \delta e^{T} P F-\delta e^{T}(D P+P D) e \frac{\dot{L}}{L}+\delta \operatorname{tr}\left(G^{T} P G\right) \\
\leq & -\delta L\|e\|^{2}+\delta\left\|e^{T} P\right\|^{2}+\delta\|F\|^{2}-\delta e^{T}(D P+P D) e \frac{\dot{L}}{L}+\delta \lambda_{\max }(P)\|G\|^{2} \\
\leq & -\delta\left(L-\|P\|^{2}\right)\|e\|^{2}+\delta\left(4 n^{2} \theta_{1}^{2}\left(\|e\|^{2}+\|z\|^{2}\right)+2 n \theta_{2}^{2}\|\eta\|^{2}\right)-\delta e^{T}(D P+P D) e \frac{\dot{L}}{L} \\
& +\delta \lambda_{\max }(P)\left(4 n^{2} \theta_{3}^{2}\left(\|e\|^{2}+\|z\|^{2}\right)+2 n \theta_{4}^{2}\|\eta\|^{2}\right) \\
\leq & -\left[\delta\left(L-\|P\|^{2}-c_{1}\right)\right]\|e\|^{2}+c_{1} \delta\|z\|^{2}+c_{2} \delta\|\eta\|^{2}
\end{aligned}
$$

where $c_{1}=4 n^{2}\left(\theta_{1}^{2}+\lambda_{\max }(P) \theta_{3}^{2}\right)$, and $c_{2}=2 n\left(\theta_{2}^{2}+\lambda_{\max }(P) \theta_{4}^{2}\right)$.

Select $V_{2}=z^{T} Q z$; then

$$
\dot{V}_{2}=z^{T} L\left(B^{T} Q+Q B\right) z+2 L z^{T} Q a e_{1}-z^{T}(D Q+Q D) z \frac{\dot{L}}{L}
$$

Notice that

$$
2 L z^{T} Q a e_{1} \leq L\|z\|^{2}+L\|Q a\|^{2} e_{1}^{2}
$$

By (4.10), we get

$$
\dot{V}_{2} \leq-L\|z\|^{2}+L\|Q a\|^{2} e_{1}^{2} .
$$


Select $V=\left(\left(\delta c_{2}+\tau\right) / \alpha\right) V_{\eta}+V_{1}+V_{2}$, where $\tau>0$; then from (3.3), (5.2), and (5.5), we can obtain that

$$
\begin{aligned}
\mathfrak{L} V & \leq-\tau\|\eta\|^{2}+\frac{\delta c_{2}+\tau}{\alpha} r y^{2}-\left[\delta\left(L-\|P\|^{2}-c_{1}\right)-L\|Q a\|^{2}\right]\|e\|^{2}-\left(L-\delta c_{1}\right)\|z\|^{2} \\
& \leq-\tau\|\eta\|^{2}+2 \frac{\delta c_{2}+\tau}{\alpha} r e_{1}^{2}+2 \frac{\delta c_{2}+\tau}{\alpha} r z_{1}^{2}-\left[\delta\left(L-\|P\|^{2}-c_{1}\right)-L\|Q a\|^{2}\right]\|e\|^{2}-\left(L-\delta c_{1}\right)\|z\|^{2} \\
& =-\tau\|\eta\|^{2}-\left[\delta\left(L-\|P\|^{2}-c_{1}\right)-L\|Q a\|^{2}-2 \frac{\delta c_{2}+\tau}{\alpha} r\right]\|e\|^{2}-\left(L-\delta c_{1}-2 \frac{\delta c_{2}+\tau}{\alpha} r\right)\|z\|^{2} .
\end{aligned}
$$

Let $\delta=\|Q a\|^{2}+1, c_{3}=\delta\|P\|^{2}+\delta c_{1}+2\left(\left(\delta c_{2}+\tau\right) / \alpha\right) r$; then

$$
\mathfrak{L} V \leq-\tau\|\eta\|^{2}-\left(L-c_{3}\right)\left(\|e\|^{2}+\|z\|^{2}\right) .
$$

The main result of the paper is summarized in the following theorem.

Theorem 5.1. Suppose that the system (3.1) satisfies Assumptions 3.1 and 3.2; then under the control law (4.7) and the observer (4.1), the closed-loop system has a unique solution on $[0, \infty)$ and the solution process is bounded almost surely; moreover, $\lim _{t \rightarrow \infty}\|x(t)\|=0$ a.s., and $\lim _{t \rightarrow \infty}\|\eta(t)\|=$ 0 a.s.

Proof. The proof process can be divided three parts.

First, we prove that $L(t)$ is bounded on $[0, \infty)$. This can be done by a contradiction argument. Suppose $\lim _{t \rightarrow \infty} L(t)=\infty$. Since $\dot{L}(t)=e_{1}^{2} \geq 0, L(t)$ is a monotone nondecreasing function. Thus, there exists a finite time $t<\infty$, such that

$$
L(t) \geq c_{3}+1, \quad \forall t \in[t, \infty)
$$

From (5.7), it follows that

$$
\mathfrak{L} V \leq-\tau\|\eta\|^{2}-\|e\|^{2}-\|z\|^{2}, \quad \forall t \in[t, \infty)
$$

With Lemma 2.2, we can obtain that

$$
\int_{t}^{\infty} e_{1}^{2} d t \leq \int_{t}^{\infty}\|e\|^{2} d t<+\infty \text { a.s. }
$$

By this and the definition of $\dot{L}(t)$, we have

$$
+\infty=L(\infty)-L(t)=\int_{t}^{\infty} \dot{L}(t) d t=\int_{t}^{\infty} e_{1}^{2} d t<+\infty \text { a.s., }
$$

which leads to a contradiction. Thus, the dynamic gain $L(t)$ is well defined and bounded on $[0, \infty)$. Moreover, from $\dot{L}(t)=e_{1}^{2}$, it is concluded that $\int_{0}^{\infty} e_{1}^{2} d t<\infty$. 
From (5.7) and the boundedness of $L(t)$, it can be proved that $\mathfrak{L} V \leq \beta V$ for a positive constant $\beta$ and for $\forall t \in[0, \infty)$. From Lemma 2.1, we can show the existence and uniqueness of global solution for the system (4.11).

Next, we claim that $z$ is well defined and bounded on the interval $[0,+\infty)$.

From (5.5) and $\dot{L}(t)=e_{1}^{2}$, one can obtain that

$$
\dot{V}_{2} \leq-L\|z\|^{2}+L\|Q a\|^{2} e_{1}^{2} \leq-\|z\|^{2}+\|Q a\|^{2} L \dot{L} .
$$

Then, for $\forall t \in[0, \infty)$, integrate two sides of the above inequality:

$$
\begin{aligned}
\lambda_{\min }(Q)\|z\|^{2}-z^{T}(0) Q z(0) & \leq V_{2}(t)-V_{2}(0) \\
& \leq-\int_{0}^{t}\|z\|^{2} d t+\int_{0}^{t}\|Q a\|^{2} L \dot{L} d t=-\int_{0}^{t}\|z\|^{2} d t+\frac{1}{2}\|Q a\|^{2}\left(L^{2}(t)-1\right) .
\end{aligned}
$$

From (5.13) we can obtain that, for $\forall t \in[0, \infty)$,

$$
\begin{gathered}
\|z\|^{2} \leq \frac{1}{\lambda_{\min }(Q)}\left(z^{T}(0) Q z(0)+\frac{1}{2}\|Q a\|^{2}\left(L^{2}(t)-1\right)\right), \\
\int_{0}^{t}\|z\|^{2} d t \leq z^{T}(0) Q z(0)+\frac{1}{2}\|Q a\|^{2}\left(L^{2}(t)-1\right) .
\end{gathered}
$$

Since $L(t)$ is bounded on $[0,+\infty)$, the previous inequalities imply the boundedness of $z$ and $\int_{0}^{\infty}\|z\|^{2} d t$.

Finally, we prove the boundedness of $e$ and $\eta$ on $[0,+\infty)$.

Let

$$
\xi_{i}=\frac{x_{i}-\widehat{x}_{i}}{\bar{L}^{i-1}}, \quad i=1, \ldots, n, \quad \xi=\left(\xi_{1}, \ldots, \xi_{n}\right)^{T},
$$

where $\bar{L}$ is a constant satisfying

$$
\bar{L}=\max \left\{3+\|P\|^{2}+c_{1}, L(+\infty)\right\} .
$$

Then

$$
d \xi=\left(\bar{L} A \xi+\bar{L} a \xi_{1}-L \Gamma a \xi_{1}+\bar{F}\right) d t+\bar{G} d w
$$

where

$$
\bar{F}=\left(f_{1} \frac{f_{2}}{\bar{L}} \cdots \frac{f_{n}}{\bar{L}^{n-1}}\right)^{T}, \quad \bar{G}=\left(g_{1} \frac{g_{2}}{\bar{L}} \cdots \frac{g_{n}}{\bar{L}^{n-1}}\right)^{T}, \quad \Gamma=\operatorname{diag}\left(1 \frac{L}{\bar{L}} \cdots \frac{L^{n-1}}{\bar{L}^{n-1}}\right) .
$$


Similar to (5.1), we have

$$
\begin{aligned}
& \|\bar{F}\|^{2} \leq \sum_{i=1}^{n}\left|\frac{f_{i}}{\bar{L}^{i-1}}\right|^{2} \leq 4 n^{2} \theta_{1}^{2}\left(\|\xi\|^{2}+\|z\|^{2}\right)+2 n \theta_{2}^{2}\|\eta\|^{2}, \\
& \|\bar{G}\|^{2} \leq \sum_{i=1}^{n}\left|\frac{g_{i}}{\bar{L}^{i-1}}\right|^{2} \leq 4 n^{2} \theta_{3}^{2}\left(\|\xi\|^{2}+\|z\|^{2}\right)+2 n \theta_{4}^{2}\|\eta\|^{2} .
\end{aligned}
$$

Now we consider the Lyapunov function $V_{3}=\xi^{T} P \xi+\left(\left(c_{2}+\tau\right) / \alpha\right) V_{\eta}$. A straightforward calculation shows that along the trajectories of (4.11), we can obtain

$$
\mathfrak{L} V_{3} \leq-\bar{L}\|\xi\|^{2}+2 \xi_{1} \bar{L} a^{T} P \xi-2 \xi_{1} L a^{T} \Gamma P \xi+2 \bar{F}^{T} P \xi+\operatorname{tr}\left(\bar{G}^{T} P \bar{G}\right)-\left(c_{2}+\tau\right)\|\eta\|^{2}+\frac{c_{2}+\tau}{\alpha} r y^{2}
$$

Noticing that

$$
\begin{aligned}
& 2 \xi_{1} \bar{L} a^{T} P \xi \leq \bar{L}^{2}\left\|a^{T} P\right\|^{2} \xi_{1}^{2}+\|\xi\|^{2}, \\
& -2 \xi_{1} L a^{T} \Gamma P \xi \leq L^{2}\left\|a^{T} \Gamma P\right\|^{2} \xi_{1}^{2}+\|\xi\|^{2}, \\
& 2 \bar{F}^{T} P \xi \leq\|P\|^{2}\|\xi\|^{2}+\|\bar{F}\|^{2} \leq\left(\|P\|^{2}+4 n^{2} \theta_{1}^{2}\right)\|\xi\|^{2}+4 n^{2} \theta_{1}^{2}\|z\|^{2}+2 n \theta_{2}^{2}\|\eta\|^{2}, \\
& \operatorname{tr}\left(\bar{G}^{T} P \bar{G}\right) \leq \lambda_{\max }(P)\|\bar{G}\|^{2} \leq 4 n^{2} \lambda_{\max }(P) \theta_{3}^{2}\left(\|\xi\|^{2}+\|z\|^{2}\right)+2 n \lambda_{\max }(P) \theta_{4}^{2}\|\eta\|^{2},
\end{aligned}
$$

we have

$$
\begin{aligned}
\mathfrak{L} V_{3} \leq & -\left(\bar{L}-2-\|P\|^{2}-c_{1}\right)\|\xi\|^{2}-\left(c_{2}+\tau\right)\|\eta\|^{2}+\left(\bar{L}^{2}\left\|a^{T} P\right\|^{2}+L^{2}\left\|a^{T} \Gamma P\right\|^{2}\right) \xi_{1}^{2} \\
& +c_{1}\|z\|^{2}+\frac{c_{2}+\tau}{\alpha} r y^{2}+c_{2}\|\eta\|^{2} \\
\leq & -\|\xi\|^{2}-\tau\|\eta\|^{2}+c_{1}\|z\|^{2}+2 \frac{c_{2}+\tau}{\alpha} r\left(e_{1}^{2}+z_{1}^{2}\right)+\bar{L}^{2}\left(\left\|a^{T} P\right\|^{2}+\left\|a^{T} \Gamma P\right\|^{2}\right) e_{1}^{2} .
\end{aligned}
$$

Since $\int_{0}^{\infty}\|z\|^{2} d t$ and $\int_{0}^{\infty} e_{1}^{2} d t$ are bounded, from Lemma 2.2, we can obtain that $\|\eta(t)\|$ and $\|\xi(t)\|$ are bounded almost surely, $\lim _{t \rightarrow \infty}\|\xi(t)\|=0$ a.s., and $\lim _{t \rightarrow \infty}\|\eta(t)\|=0$ a.s. From the definition of $e$ and $\xi$, we have that $\|e(t)\|$ is bounded almost surely and $\lim _{t \rightarrow \infty}\|e(t)\|=0$ a.s. Moreover, $\lim _{t \rightarrow \infty}\|z(t)\|=0$ a.s. is followed from (5.5); then it leads to $\lim _{t \rightarrow \infty}\|x(t)\|=0$ a.s.. 


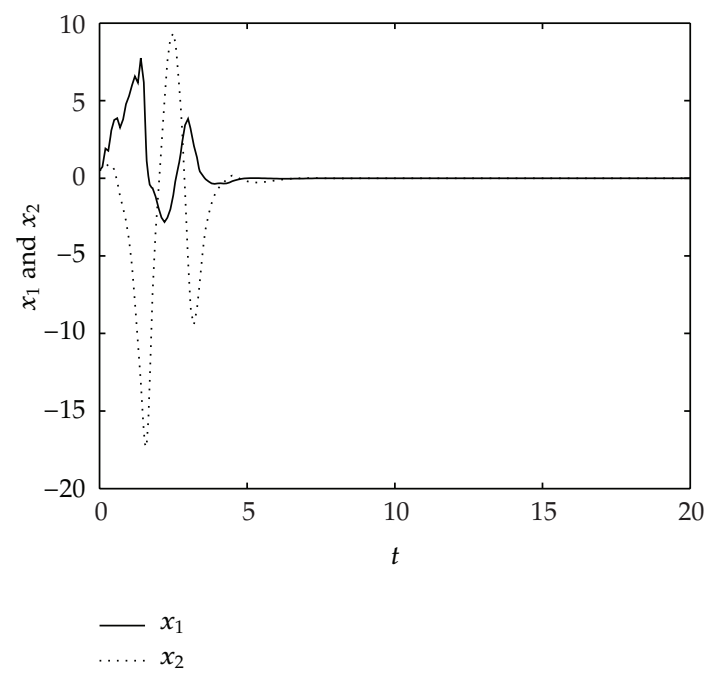

Figure 1: System states $x_{1}$ and $x_{2}$.

Thus, we obtain that $\lim _{t \rightarrow \infty}\|\eta(t)\|=0$ a.s., and $\lim _{t \rightarrow \infty}\|x(t)\|=0$ a.s. Up to now, the proof is completed.

\section{Simulation}

Considering the following stochastic nonlinear system:

$$
\begin{aligned}
d \eta & =\left(-\eta+\lambda_{1} y\right) d t+\lambda_{2} y \sin y d w \\
d x_{1} & =\left(x_{2}+\eta+\lambda_{3} y\right) d t+\frac{\lambda_{4} x_{1}^{2}}{1+x_{1}^{2}} \eta d w \\
d x_{2} & =\left(u+\eta+\lambda_{5} x_{2}\right) d t \\
y & =x_{1}
\end{aligned}
$$

where $w$ is an $m$-dimensional standard Wiener process, and $\lambda_{i}, i=1, \ldots, 5$ are unknown parameters.

By Theorem 5.1, we can design the output feedback controller as follows:

$$
\begin{aligned}
d \widehat{x}_{1} & =\left(\widehat{x}_{2}+L a_{1}\left(y-\widehat{x}_{1}\right)\right) d t, \\
d \widehat{x}_{2} & =\left(u+L^{2} a_{2}\left(y-\widehat{x}_{1}\right)\right) d t, \\
\dot{L} & =\left(y-\widehat{x}_{1}\right)^{2} \\
u & =-\left(L^{2} b_{1} \widehat{x}_{1}+L b_{2} \widehat{x}_{2}\right) .
\end{aligned}
$$




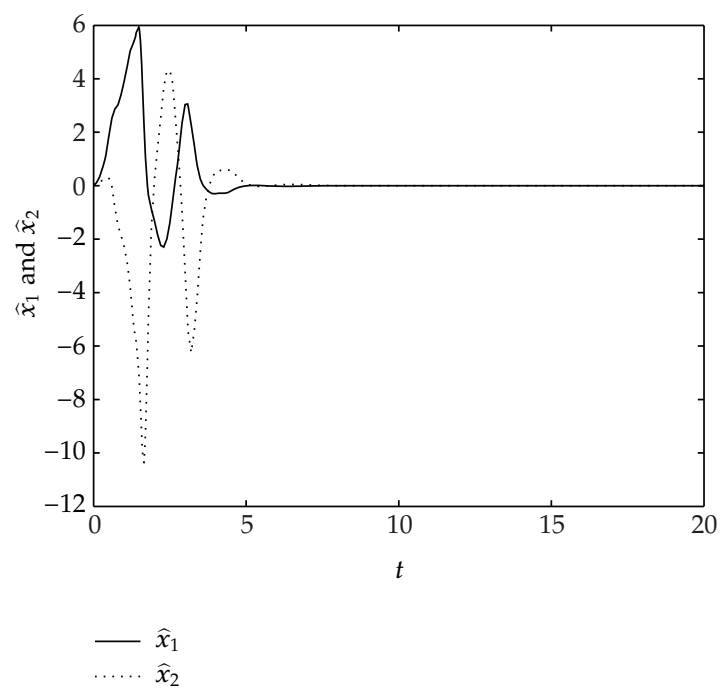

Figure 2: Observer states $\widehat{x}_{1}$ and $\widehat{x}_{2}$.

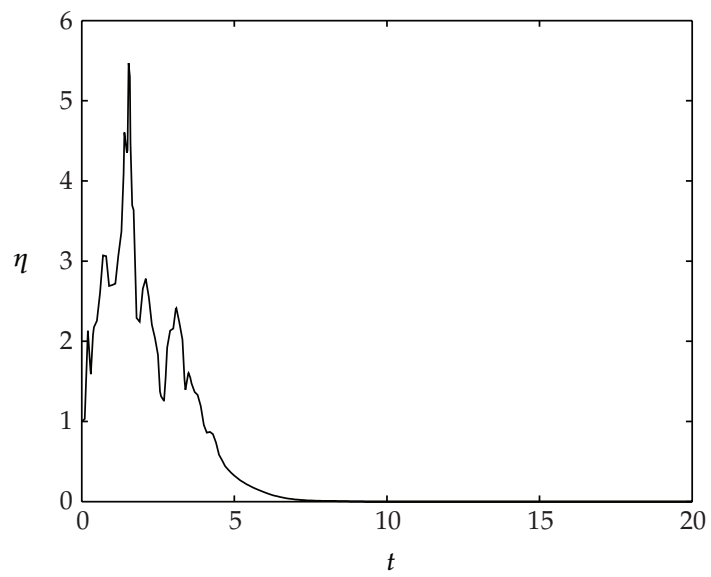

Figure 3: System states $\eta$.

The initial values of the states are $\eta(0)=1, x(0)=(0.5,0.5)^{T}, \widehat{x}(0)=(0,0)^{T}$, and $\lambda_{1}=1$, $\lambda_{2}=0.5, \lambda_{3}=0.6, \lambda_{4}=0.6$, and $\lambda_{5}=0.5$. Choosing the design parameters $a_{1}=2, a_{2}=1$, $b_{1}=1$, and $b_{2}=2$, we can obtain Figures $1,2,3,4$, and 5 . From the figures, we can see that $L$ is bounded and $\eta, x$, and $\hat{x}$ converge to zero almost surely.

\section{Conclusion}

In this paper, the adaptive output feedback control has been considered for a class of stochastic nonlinear systems with stochastic inverse dynamic and linearly bounded unmeasurable states. Like [20], a quadratic Lyapunov function is adopted instead of a quartic function 


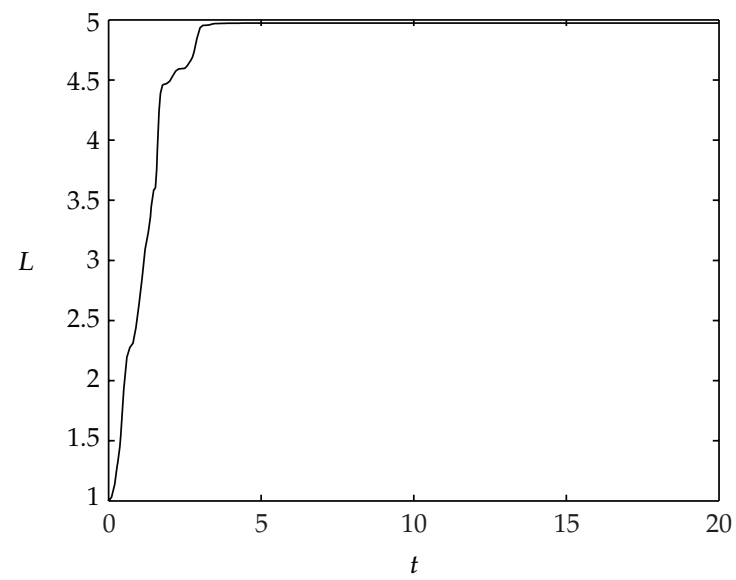

Figure 4: Dynamic gain $L$.

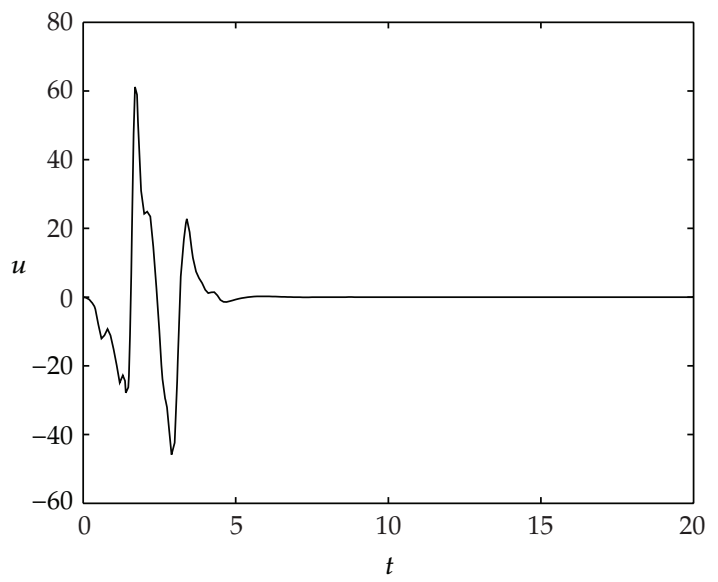

Figure 5: Control $u$.

and this makes the design process simple. Under some weak conditions, an adaptive highgain observer has been designed and the closed-loop system is globally stable in probability. Moreover, the states of the closed-loop system converge to zero almost surely.

\section{Acknowledgments}

This work is partially supported by the National Natural Science Foundation under Grant no. 61074040, Natural Science Foundation of Shandong Province under Grant no. ZR2009GL009, China Postdoctoral Science Foundation under Grant no. 20110491559, Shandong, Province Postdoctoral Innovation Foundation under Grant no. 201003041, Research Startup Foundation of Qufu Normal University and Research Foundation of Qufu Normal University under Grant no. XJ200846. 


\section{References}

[1] Y.-G. Liu and J.-F. Zhang, "Practical output-feedback risk-sensitive control for stochastic nonlinear systems with stable zero-dynamics," SIAM Journal on Control and Optimization, vol. 45, no. 3, pp. 885926, 2006.

[2] S.-J. Liu, J.-F. Zhang, and Z.-P. Jiang, “Decentralized adaptive output-feedback stabilization for largescale stochastic nonlinear systems," Automatica, vol. 43, no. 2, pp. 238-251, 2007.

[3] H. Deng and M. Krstić, "Output-feedback stabilization of stochastic nonlinear systems driven by noise of unknown covariance," Systems $\mathcal{E}$ Control Letters, vol. 39, no. 3, pp. 173-182, 2000.

[4] Z. Pan, Y. Liu, and S. Shi, “Output feedback stabilization for stochastic nonlinear systems in observer canonical form with stable zero-dynamics," Science in China F, vol. 44, no. 4, pp. 292-308, 2001.

[5] G. Arslan and T. Başar, "Risk-sensitive adaptive trackers for strict-feedback systems with output measurements," IEEE Transactions on Automatic Control, vol. 47, no. 10, pp. 1754-1758, 2002.

[6] Y. Liu, Z. Pan, and S. Shi, "Output feedback control design for strict-feedback stochastic nonlinear systems under a risk-sensitive cost," IEEE Transactions on Automatic Control, vol. 48, no. 3, pp. 509513, 2003.

[7] Y. Liu, J. Zhang, and Z. Pan, "Design of satisfaction output feedback controls for stochastic nonlinear systems under quadratic tracking risk-sensitive index," Science in China F, vol. 46, no. 2, pp. 126-144, 2003.

[8] H. Deng and M. Krstić, “Output-feedback stochastic nonlinear stabilization," IEEE Transactions on Automatic Control, vol. 44, no. 2, pp. 328-333, 1999.

[9] F. Mazenc, L. Praly, and W. P. Dayawansa, "Global stabilization by output feedback: examples and counterexamples," Systems \& Control Letters, vol. 23, no. 2, pp. 119-125, 1994.

[10] Z.-P. Jiang, "Global output feedback control with disturbance attenuation for minimum-phase nonlinear systems," Systems E Control Letters, vol. 39, no. 3, pp. 155-164, 2000.

[11] P. Krishnamurthy, F. Khorrami, and Z. P. Jiang, "Global output feedback tracking for nonlinear systems in generalized output-feedback canonical form," IEEE Transactions on Automatic Control, vol. 47, no. 5, pp. 814-819, 2002.

[12] C. Qian and W. Lin, "Output feedback control of a class of nonlinear systems: a nonseparation principle paradigm," IEEE Transactions on Automatic Control, vol. 47, no. 10, pp. 1710-1715, 2002.

[13] W. Lin, C. Qian, and X. Huang, "Disturbance attenuation of a class of non-linear systems via output feedback," International Journal of Robust and Nonlinear Control, vol. 13, no. 15, pp. 1359-1369, 2003.

[14] L. Praly, "Asymptotic stabilization via output feedback for lower triangular systems with output dependent incremental rate," IEEE Transactions on Automatic Control, vol. 48, no. 6, pp. 1103-1108, 2003.

[15] L. Praly and Z. P. Jiang, "Linear output feedback with dynamic high gain for nonlinear systems," Systems \& Control Letters, vol. 53, no. 2, pp. 107-116, 2004.

[16] S. J. Liu and J. F. Zhang, "Global output feedback stabilization for stochastic nonlinear systems with stochastic input-to-state stable zero-dynamics," in Proceedings of the 24th Chinese Control Conference, pp. 93-98, Guangzhou, China, 2005.

[17] Z.-J. Wu, X.-J. Xie, and S.-Y. Zhang, "Adaptive backstepping controller design using stochastic smallgain theorem," Automatica, vol. 43, no. 4, pp. 608-620, 2007.

[18] X. Yu and X.-J. Xie, "Output feedback regulation of stochastic nonlinear systems with stochastic iISS inverse dynamics," IEEE Transactions on Automatic Control, vol. 55, no. 2, pp. 304-320, 2010.

[19] X. Yu, X.-J. Xie, and Y.-Q. Wu, "Further results on output-feedback regulation of stochastic nonlinear systems with SiISS inverse dynamics," International Journal of Control, vol. 83, no. 10, pp. 2140-2152, 2010.

[20] S.-J. Liu and J.-F. Zhang, "Output-feedback control of a class of stochastic nonlinear systems with linearly bounded unmeasurable states," International Journal of Robust and Nonlinear Control, vol. 18, no. 6, pp. 665-687, 2008.

[21] H. Lei and W. Lin, "Universal adaptive control of nonlinear systems with unknown growth rate by output feedback," Automatica, vol. 42, no. 10, pp. 1783-1789, 2006.

[22] N. Duan, X.-J. Xie, and X. Yu, "State feedback stabilization of stochastic nonlinear systems with SiISS inverse dynamics," International Journal of Robust and Nonlinear Control, vol. 21, no. 16, pp. 1903-1919, 2011.

[23] Z.-J. Wu, X.-J. Xie, P. Shi, and Y.-Q. Xia, “Backstepping controller design for a class of stochastic nonlinear systems with Markovian switching," Automatica, vol. 45, no. 4, pp. 997-1004, 2009. 
[24] N. Duan and X.-J. Xie, "Further results on output-feedback stabilization for a class of stochastic nonlinear systems," IEEE Transactions on Automatic Control, vol. 56, no. 5, pp. 1208-1213, 2011.

[25] X. Yu, X. J. Xie, and N. Duan, "Small-gain control method for stochastic nonlinear systems with stochastic iISS inverse dynamics," Automatica, vol. 46, no. 11, pp. 1790-1798, 2010.

[26] N. Duan, X. Yu, and X.-J. Xie, "Output feedback control using small-gain conditions for stochastic nonlinear systems with SiISS inverse dynamics," International Journal of Control, vol. 84, no. 1, pp. 47-56, 2011.

[27] X.-J. Xie, N. Duan, and X. Yu, "State-feedback control of high-order stochastic nonlinear systems with SiISS inverse dynamics," IEEE Transactions on Automatic Control, vol. 56, no. 8, pp. 1921-1926, 2011.

[28] S.-J. Liu, S. S. Ge, and J.-F. Zhang, "Adaptive output-feedback control for a class of uncertain stochastic non-linear systems with time delays," International Journal of Control, vol. 81, no. 8, pp. 1210-1220, 2008.

[29] X. Mao, "Stochastic versions of the LaSalle theorem," Journal of Differential Equations, vol. 153, no. 1, pp. 175-195, 1999.

[30] X. Mao, "Some contributions to stochastic asymptotic stability and boundedness via multiple Lyapunov functions," Journal of Mathematical Analysis and Applications, vol. 260, no. 2, pp. 325-340, 2001. 


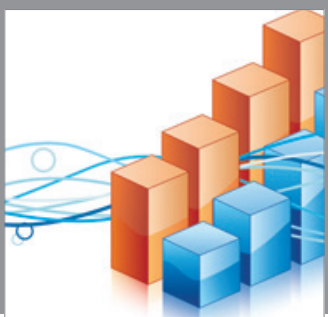

Advances in

Operations Research

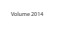

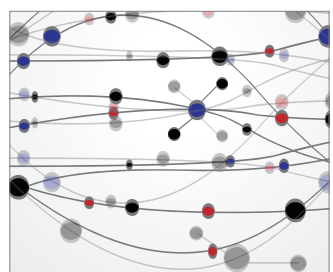

\section{The Scientific} World Journal
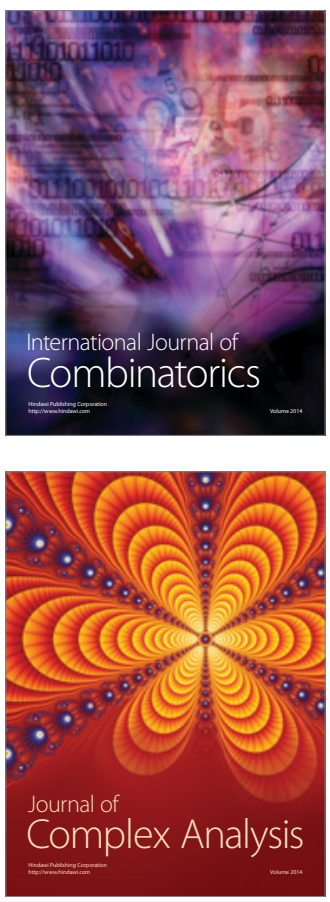

International Journal of

Mathematics and

Mathematical

Sciences
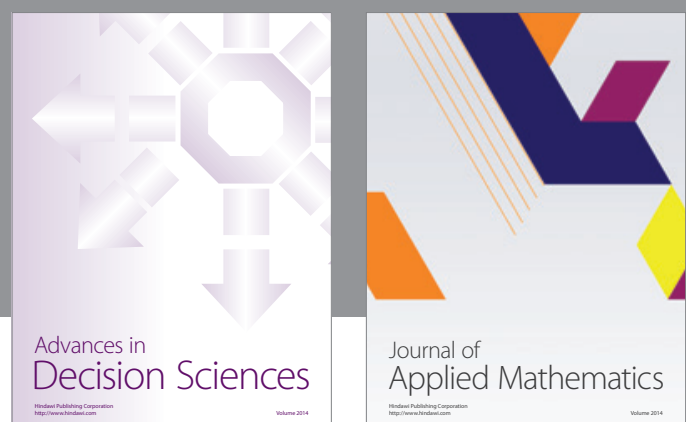

Journal of

Applied Mathematics
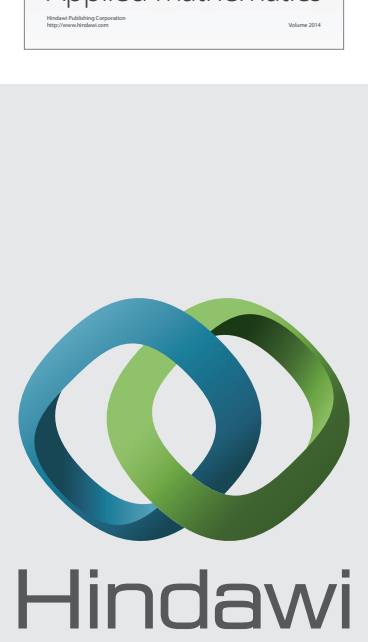

Submit your manuscripts at http://www.hindawi.com
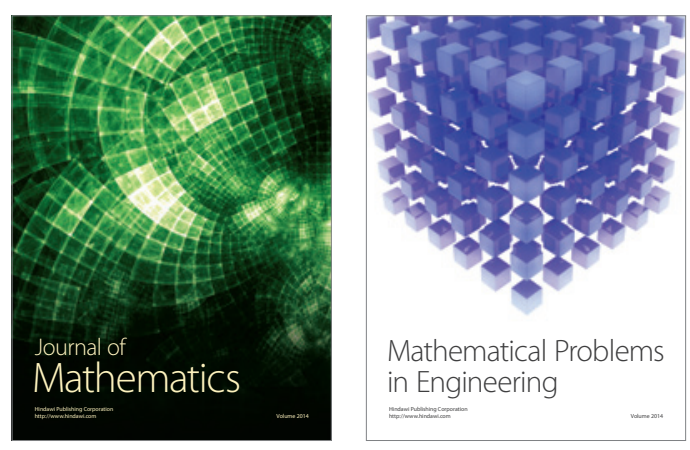

Mathematical Problems in Engineering
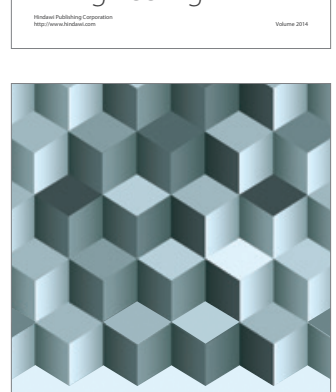

Journal of

Function Spaces
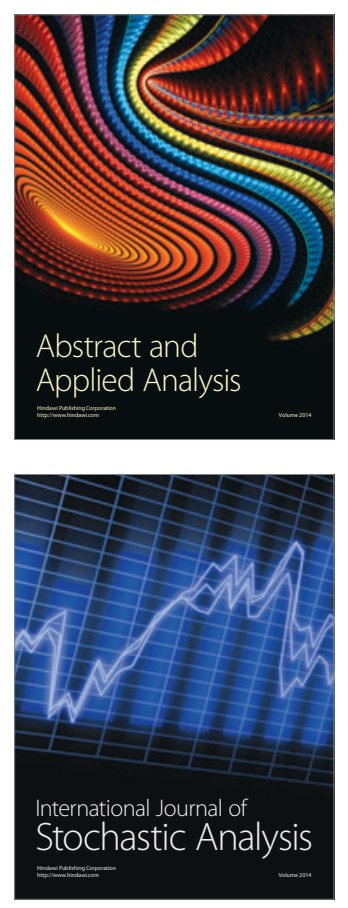

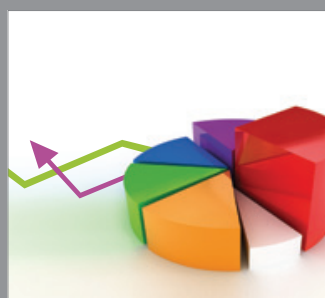

ournal of

Probability and Statistics

Promensencen
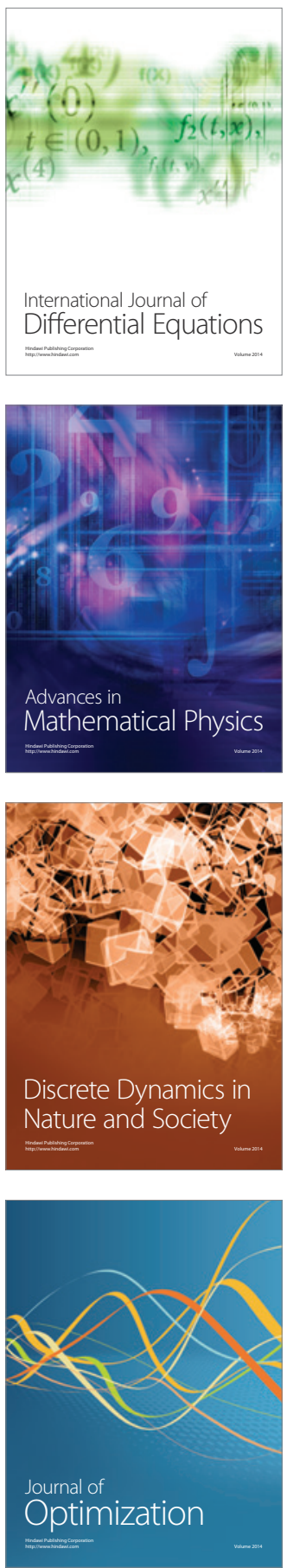